\title{
Racial/ethnic differences in the outcomes of patients with metastatic breast cancer: contributions of demographic, socioeconomic, tumor and metastatic characteristics
}

\author{
Jin-Xiao Ren ${ }^{1,2} \cdot$ Yue Gong ${ }^{1,2} \cdot$ Hong Ling $^{1} \cdot$ Xin Hu$^{1} \cdot$ Zhi-Ming Shao ${ }^{1,2,3}$ (I) \\ Received: 1 April 2018 / Accepted: 3 September 2018 / Published online: 6 October 2018 \\ (c) The Author(s) 2018
}

\begin{abstract}
Purpose Population-based estimates of racial disparities in metastatic breast cancer are lacking. We quantified the contributions of demographic, socioeconomic, tumor, and metastatic characteristics to racial differences in metastatic breast cancer and characterized the most disproportional subgroup.

Methods Patients diagnosed with metastatic breast cancer between 2010 and 2014 were identified using the Surveillance, Epidemiology, and End Results database. A multivariable Cox proportional hazards model was used to adjust each set of variables. The excess relative risk of cancer-specific and all-cause death in non-Hispanic black (NHB) versus non-Hispanic white women diagnosed with metastatic breast cancer was expressed as a percentage and was stratified by the age at diagnosis. Results We identified 13,066 female patients. NHB women exhibited substantially higher morbidity and mortality than women of other races/ethnicities. The greatest excess mortality risk for NHB women was observed in the young-onset group (18-49 years; hazard ratio: 1.57), followed by the middle-age group (50-64 years; hazard ratio: 1.42); the trend was not significant among the elderly group. Socioeconomic factors stably explained one-half of the excess risk, whereas the contribution of tumor characteristics obviously decreased with age (18-49 years, 40.7\%; 50-64 years, 33.9\%), and the metastatic pattern accounted for approximately one-tenth of the excess risk. Additionally, the disproportional death burden of NHB women persisted in less aggressive subgroups.

Conclusions By providing a comprehensive assessment of racial differences in the incidence and outcomes of patients with metastatic breast cancer, we urge the implementation of targeted preventive efforts in both the public health and clinical arenas.
\end{abstract}

Keywords Metastatic breast cancer $\cdot$ Racial disparity $\cdot$ Breast cancer prognosis $\cdot$ Age group

\section{Introduction}

Jin-Xiao Ren and Yue Gong contributed equally and share co-first authorship.

\section{Electronic supplementary material The online version of this} article (https://doi.org/10.1007/s10549-018-4956-y) contains supplementary material, which is available to authorized users.

$\mathrm{Xin} \mathrm{Hu}$

xinhu@fudan.edu.com

$\checkmark$ Zhi-Ming Shao

zhi_ming_shao@163.com

1 Department of Breast Surgery, Key Laboratory of Breast Cancer in Shanghai, Cancer Institute, Fudan University Shanghai Cancer Center, Fudan University, Shanghai 200032, China
The presence of distant metastases at initial diagnosis represents an important cause of morbidity and mortality among all patients with breast neoplasms, and the burden on the population is unequal. Metastatic breast cancer represents 9\% of diagnoses among non-Hispanic black (NHB) women

2 Department of Oncology, Shanghai Medical College, Fudan University, 270 Dong-An Road, Shanghai 200032, China

3 Institutes of Biomedical Science, Fudan University, Shanghai 200032, China 
compared with 5-6\% of diagnoses in other racial/ethnic groups. Regarding the survival gains in patients with distantstage disease from 1975 to 2013, the 5-year cause-specific survival of non-Hispanic white (NHW) women (19-37\%) was higher than that of other racial/ethnic groups, particularly NHB women (16-26\%) [1, 2]. Racial disparities in breast cancer have been well documented but estimates of racial variances in metastatic breast cancer are lacking.

Previous research on racial differences in all-stage or early-stage breast cancer has attributed the disproportionate incidences of these diseases to characteristics at diagnosis, such as age, socioeconomic status (SES), and tumor biology [3-7]. A recent study examined the black-white disparity in non-elderly women diagnosed with stage I to III breast cancer from 2004 to 2013 in the National Cancer Data Base, finding that differences in insurance coverage accounted for one-third of the survival disparity, whereas differences in tumor characteristics accounted for one-fifth of the disparity [8]. According to another recent study on patients with all-stage breast cancer conducted using only California Cancer Registry data, the stage at diagnosis accounted for $24 \%$ of the differences in cancer-specific survival, whereas hormone receptor (HoR) status accounted for $9 \%$ and neighborhood SES accounted for 6\% [9]. A recent review concluded that racial differences in the stage at diagnosis were not significant but rather gained importance by highlighting differences in tumor biology, patterns of care and other prognostic factors [10]. Here, we estimate stage-specific racial disparities in metastatic breast cancer and examine the contributions of relevant prognostic factors. In addition, the metastatic pattern, which has been reported to display a significantly correlation with the prognosis of metastatic breast cancer, has not yet been investigated in studies using mediating models for racial disparities.

The primary purpose of this study was to quantitatively estimate the contributions of demographic, socioeconomic, tumor and metastatic characteristics to racial differences in metastatic breast cancer using the Surveillance, Epidemiology, and End Results (SEER) database at the population level. We also sought to characterize independent clinical predictors of the variances in survival by race in patients with metastatic breast cancer to improve prevention through suitable clinical strategies and public interventions.

\section{Methods}

\section{Data source}

We obtained population-based data from the SEER 18 registry research database. SEER covers approximately $27.8 \%$ of the US population, including $24.9 \%$ of Whites, $25.6 \%$ of African Americans, $38.4 \%$ of Hispanics, $30.6 \%$ of American
Indians and Alaskan Natives, 50.4\% of Asians, and 66.5\% of Hawaiian/Pacific Islanders (based on the 2010 Census) [11]. The data reported in this study represent the most recent follow-up (November 2017 Submission) available in the SEER database [12].

\section{Cohort selection}

We identified 17,579 female adults (aged $>18$ years) who were diagnosed with adjusted American Joint Committee on Cancer (AJCC) seventh edition stage IV breast cancer between 2010 and 2014. This time frame was selected because information on distant metastases to specific sites and molecular subtypes at the time of the initial cancer diagnosis was available only for patients who were diagnosed after 2010. We excluded patients with a previous cancer diagnosis $(n=3321)$; patients diagnosed at autopsy or on a death certificate $(n=13)$; patients lacking histological confirmation $(n=871)$; patients with missing follow-up data $(n=266)$; and patients whose race/ethnicity was not available $(n=42)$. Our final cohort included 13,066 women, comprising $74.3 \%$ of all 17,579 previously identified patients (Fig. 1).

\section{Covariate and outcome measures}

Our primary outcome of interest was vital status, including follow-up time, breast cancer-specific survival (BCSS), overall survival (OS), and excess relative risk (ERR) of death among different racial groups (versus NHW). The follow-up time was defined as the time between the date of diagnosis and the date of death or the last follow-up visit. BCSS was defined as the interval from the date of diagnosis to the date of death due to breast cancer. OS was defined as the interval from the date of diagnosis to the date of death from any cause.

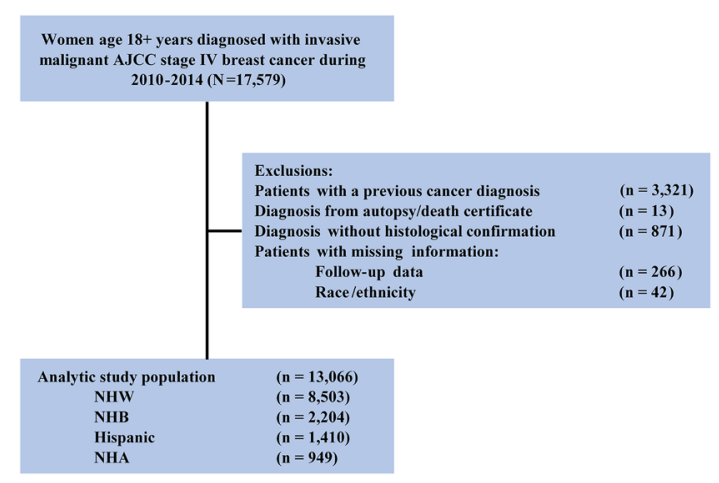

Fig. 1 Schematic representation of the patient selection process. AJCC American Joint Committee on Cancer, NHA non-Hispanic Asian or Pacific Islander/American Indian/Alaskan Native, $N H B$ nonHispanic black, $N H W$ non-Hispanic white 
Race/ethnicity in the SEER database was classified into 5 major groups, including NHW, NHB, non-Hispanic Asian or Pacific Islander (NHAPI), non-Hispanic American Indian/ Alaska Native (NHAIAN), and Hispanic (all races). Given the small number of NHAPI and NHAIAN women, we merged the two groups into one. Thus, we classified race/ ethnicity into four mutually exclusive groups: (1) NHW, (2) NHB, (3) Hispanic (all races), and (4) Non-Hispanic Asian or Pacific Islander/American Indian/Alaskan Native (NHA).

Neighborhood SES is a county-level, time-dependent composite index developed through principal components analysis of census tract data on education, occupation, employment, household income, poverty, rent and house value. The composite SES score is categorized into quintiles with roughly equal populations, ranging from lowest to highest SES [13, 14].

\section{Statistical analyses}

The incidence and mortality rates were standardized to the 2000 US standard population by age and were expressed per 100,000 persons, as calculated using NCI SEER*Stat software (version 8.3.5) [12, 15].

Kruskal-Wallis tests and Pearson's $\chi^{2}$ tests were employed to compare the characteristics of patients stratified by racial/ ethnic group for continuous and categorical variables, respectively. Detailed classification information is presented in Table 1. Patients with missing data for a given variable were excluded from the comparative analysis. Univariate and multivariate Cox proportional hazards models were used to identify prognostic factors for OS in the cohort patients. As a non-trivial proportion of the patients died from causes other than breast cancer in the study cohort, Fine and Gray's competing risk model was employed to compare BCSS [16, 17]. The variables employed for adjustment were modeled as covariates to estimate the contribution of each factor or set of factors to racial disparity. Model one was the unadjusted model. Demographics (age at diagnosis), SES (individuallevel insurance and marital status, together with area-level neighborhood SES), tumor characteristics (histological type, grade, tumor size, number of positive regional lymph nodes, and molecular subtype) and metastatic patterns (number and site of distant metastases) were adjusted in models two to five, respectively. Finally, model six adjusted for all variables. Hazard ratios (HRs) and associated 95\% confidence intervals (CIs) were estimated from both the BCSS and OS for each model. Next, the ERR of death was calculated by subtracting one from the HR. Finally, the proportion of total ERR explained by each set of variables, as well as by all variables combined, was calculated. Analyses of subgroups stratified according to baseline characteristics were used to evaluate the consistency of racial disparities in survival after adjusting for all other prognostic factors.
The significance level of the $P$ value was set to 0.05 for a two-tailed analysis. Statistical analyses were performed using R version 3.3.3 (R Foundation for Statistical Computing, Vienna, Austria) and SPSS version 22.0 (SPSS, Inc., Chicago, IL).

\section{Results}

\section{Incidence and mortality rates of metastatic breast cancer among women}

The incidence and mortality rates (2010-2014) of metastatic breast cancer among women varied substantially by race/ ethnicity in the United States (Fig. 2a). NHB women had exhibited higher incidence and mortality rates than women of other races/ethnicities, whereas NHA women exhibited the lowest incidence and mortality rates. The disparities in the morbidity and mortality of metastatic breast cancer among races/ethnicities remained relatively stable between 2005 and 2014 (the most recent 10 years of data available) (Fig. S1A-B) as well as in trends stratified by HoR status (Fig. S1C-D). The trends in the incidence of HoR + metastatic breast cancer (2005-2014) among the groups stratified by race/ethnicity (Fig. S1C) showed a significant increase among NHW (4.3\% per year), NHB (4.8\% per year), and NHA women (3.5\% per year), but a stable trend among Hispanic women. Racial differences in the incidence and mortality rates of metastatic breast cancer varied according to age (Fig. 2b, c). The incidence rate of distant-stage breast cancer in NHW women was parallel to that of Hispanic or NHA women before the age of 50 years but was much higher thereafter. NHA women displayed lower rates and a better prognosis after the age of 65 years. NHB women were more likely to die from breast cancer at any age.

\section{Patient characteristics}

Of the 13,066 women with metastatic breast cancer at the time of diagnosis, 8503 (65.1\%) were NHW; 2204 (16.9\%) were NHB; 1410 (10.8\%) were Hispanic; and 949 (7.3\%) were NHA (Table 1). The median length of follow-up was 19 months (mean, 22.6 months). Overall, patient characteristics were significantly different among the different racial groups. Compared with NHW women, Hispanic women were, on average, 8 years younger at presentation (63 vs. 55 years) and were more likely to have Medicaid insurance ( $37.2 \%$ vs. $14.2 \%$ ); NHB women were more likely to have poor socioeconomic support (11.2\% vs. $5.5 \%$ for uninsured; $67.6 \%$ vs. $48.5 \%$ for unmarried; $39.8 \%$ vs. $12.3 \%$ for lowest SES neighborhoods); NHB women were also substantially more likely to present with high-grade tumors $(47.6 \%$ vs. $36.9 \%)$ with positivity of regional lymph nodes $(73.7 \%$ vs. 
Table 1 Demographic and clinicopathological characteristics of patients stratified by race/ethnicity

\begin{tabular}{|c|c|c|c|c|c|c|}
\hline Characteristic & $\begin{array}{l}\text { Total } \\
n=13,066(\%)\end{array}$ & $\begin{array}{l}\text { NHW } \\
n=8503(\%)\end{array}$ & $\begin{array}{l}\text { NHB } \\
n=2204(\%)\end{array}$ & $\begin{array}{l}\text { Hispanic } \\
n=1410(\%)\end{array}$ & $\begin{array}{l}\text { NHA } \\
n=949(\%)\end{array}$ & $P$ value $^{\mathrm{a}}$ \\
\hline \multicolumn{7}{|c|}{ Age at diagnosis, years } \\
\hline Median (IQR) & $61(51-71)$ & $63(54-73)$ & $58(49-67)$ & $55(46-65)$ & $57(48-65)$ & $<0.001$ \\
\hline \multicolumn{7}{|l|}{ Insurance status } \\
\hline Insured & $9539(73.0)$ & $6827(80.3)$ & $1306(59.3)$ & $766(54.3)$ & $640(67.4)$ & \multirow[t]{3}{*}{$<0.001$} \\
\hline Medicaid & $2636(20.2)$ & $1209(14.2)$ & $652(29.6)$ & $524(37.2)$ & $251(26.4)$ & \\
\hline Uninsured & $891(6.8)$ & $467(5.5)$ & $246(11.2)$ & $120(8.5)$ & $58(6.1)$ & \\
\hline \multicolumn{7}{|l|}{ Marital status } \\
\hline Married & $5552(42.5)$ & $3896(45.8)$ & $560(25.4)$ & $605(42.9)$ & $491(51.7)$ & \multirow[t]{3}{*}{$<0.001$} \\
\hline Not married ${ }^{b}$ & $6751(51.7)$ & $4123(48.5)$ & $1490(67.6)$ & $735(52.1)$ & $403(42.5)$ & \\
\hline Unknown & $763(5.8)$ & $484(5.7)$ & $154(7.0)$ & $70(5.0)$ & $55(5.8)$ & \\
\hline \multicolumn{7}{|c|}{ Neighborhood SES } \\
\hline Q1 (low) & $2515(19.2)$ & $1045(12.3)$ & $877(39.8)$ & $450(31.9)$ & $143(15.1)$ & \multirow[t]{6}{*}{$<0.001$} \\
\hline $\mathrm{Q} 2$ & $2624(20.1)$ & $1583(18.6)$ & $524(23.8)$ & $332(23.5)$ & 185 (19.5) & \\
\hline Q3 & $2747(21.0)$ & $1898(22.3)$ & $354(16.1)$ & $287(20.4)$ & 208 (21.9) & \\
\hline Q4 & 2596 (19.9) & $1954(23.0)$ & $256(11.6)$ & $183(13.0)$ & 203 (21.4) & \\
\hline Q5 (high) & 2419 (18.5) & 1918 (22.6) & $162(7.4)$ & $141(10.0)$ & 198 (20.9) & \\
\hline Missing & $165(1.3)$ & $105(1.2)$ & $31(1.4)$ & $17(1.2)$ & $12(1.3)$ & \\
\hline \multicolumn{7}{|l|}{ Histological type } \\
\hline IDC & $8782(67.2)$ & $5551(65.0)$ & $1576(71.5)$ & $959(68.0)$ & $696(73.3)$ & \multirow[t]{3}{*}{$<0.001$} \\
\hline ILC & $1372(10.5)$ & 992 (11.7) & $174(7.9)$ & $141(10.0)$ & $65(6.8)$ & \\
\hline Others & $2912(22.3)$ & $1960(23.1)$ & 454 (20.6) & $310(22.0)$ & 188 (19.8) & \\
\hline \multicolumn{7}{|l|}{ Grade } \\
\hline $\mathrm{I} / \mathrm{II}$ & $5005(38.3)$ & $3442(40.5)$ & $696(31.6)$ & $516(36.6)$ & $351(37.0)$ & \multirow[t]{3}{*}{$<0.001$} \\
\hline III/UD & $5230(40.0)$ & 3137 (36.9) & $1050(47.6)$ & $625(44.3)$ & $418(44.0)$ & \\
\hline Unknown & $2831(21.7)$ & $1924(22.6)$ & $458(20.8)$ & $269(19.1)$ & $180(19.0)$ & \\
\hline \multicolumn{7}{|l|}{ Tumor size (mm) } \\
\hline $0-20$ & 1907 (14.6) & $1342(15.8)$ & $281(12.7)$ & $185(13.1)$ & $99(10.4)$ & \multirow[t]{4}{*}{$<0.001$} \\
\hline $21-50$ & 4743 (36.3) & 3201 (37.6) & $713(32.4)$ & 483 (34.3) & $346(36.5)$ & \\
\hline$>50$ & $3903(29.9)$ & $2307(27.1)$ & $771(35.0)$ & $490(34.8)$ & $335(35.3)$ & \\
\hline Unknown & $2513(19.2)$ & $1653(19.4)$ & 439 (19.9) & $252(17.9)$ & $169(17.8)$ & \\
\hline \multicolumn{7}{|c|}{ Regional lymph node positive } \\
\hline No & $2860(21.9)$ & $2040(24.0)$ & 395 (17.9) & $268(19.0)$ & $157(16.5)$ & \multirow[t]{3}{*}{$<0.001$} \\
\hline Yes & $8803(67.4)$ & $5474(64.4)$ & $1624(73.7)$ & 1009 (71.6) & $696(73.3)$ & \\
\hline Unknown & $1403(10.7)$ & $989(11.6)$ & $185(8.4)$ & $133(9.4)$ & $96(10.1)$ & \\
\hline \multicolumn{7}{|c|}{ Molecular subtype } \\
\hline HER2-/HoR + & $6866(52.5)$ & $4668(54.9)$ & $1004(45.6)$ & $695(49.3)$ & $499(52.6)$ & \multirow[t]{5}{*}{$<0.001$} \\
\hline HER2+/HoR+ & $1930(14.8)$ & $1211(14.2)$ & $336(15.2)$ & $233(16.5)$ & $150(15.8)$ & \\
\hline HER2 +/HoR- & $1056(8.1)$ & $623(7.3)$ & $185(8.4)$ & $136(9.6)$ & $112(11.8)$ & \\
\hline HER2-/HoR- & $1612(12.3)$ & 927 (10.9) & 416 (18.9) & $170(12.1)$ & $99(10.4)$ & \\
\hline Unknown & $1602(12.3)$ & $1074(12.6)$ & $263(11.9)$ & $176(12.5)$ & $89(9.4)$ & \\
\hline \multicolumn{7}{|c|}{ No. of distant metastases } \\
\hline 1 & $6806(52.1)$ & $4535(53.3)$ & $1106(50.2)$ & $708(50.2)$ & $457(48.2)$ & \multirow[t]{3}{*}{$<0.001$} \\
\hline$>1$ & $5356(41.0)$ & 3367 (39.6) & $974(44.2)$ & $594(42.1)$ & $421(44.4)$ & \\
\hline Unknown & $904(6.9)$ & $601(7.1)$ & $124(5.6)$ & $108(7.7)$ & $71(7.5)$ & \\
\hline \multicolumn{7}{|l|}{ Bone } \\
\hline Yes & $8515(65.2)$ & $5720(67.3)$ & $1315(59.7)$ & $884(62.7)$ & $596(62.8)$ & \multirow[t]{3}{*}{$<0.001$} \\
\hline No & $4245(32.5)$ & $2576(30.3)$ & $848(38.5)$ & $494(35.0)$ & $327(34.5)$ & \\
\hline Unknown & $306(2.3)$ & $207(2.4)$ & $41(1.9)$ & $32(2.3)$ & $26(2.7)$ & \\
\hline
\end{tabular}


Table 1 (continued)

\begin{tabular}{|c|c|c|c|c|c|c|}
\hline Characteristic & $\begin{array}{l}\text { Total } \\
n=13,066(\%)\end{array}$ & $\begin{array}{l}\text { NHW } \\
n=8503(\%)\end{array}$ & $\begin{array}{l}\text { NHB } \\
n=2204(\%)\end{array}$ & $\begin{array}{l}\text { Hispanic } \\
n=1410(\%)\end{array}$ & $\begin{array}{l}\text { NHA } \\
n=949(\%)\end{array}$ & $P$ value $^{\mathrm{a}}$ \\
\hline \multicolumn{7}{|l|}{ Brain } \\
\hline Yes & $938(7.2)$ & $578(6.8)$ & $184(8.3)$ & 112 (7.9) & $64(6.7)$ & 0.036 \\
\hline No & $11,567(88.5)$ & $7543(88.7)$ & $1946(88.3)$ & $1232(87.4)$ & $846(89.1)$ & \\
\hline Unknown & $561(4.3)$ & $382(4.5)$ & $74(3.4)$ & $66(4.7)$ & $39(4.1)$ & \\
\hline \multicolumn{7}{|l|}{ Liver } \\
\hline Yes & $3412(26.1)$ & $2184(25.7)$ & $628(28.5)$ & $338(24.0)$ & $262(27.6)$ & 0.030 \\
\hline No & $9218(70.5)$ & $6033(71.0)$ & $1513(68.6)$ & $1016(72.1)$ & $656(69.1)$ & \\
\hline Unknown & $436(3.3)$ & $286(3.4)$ & $63(2.9)$ & $56(4.0)$ & $31(3.3)$ & \\
\hline \multicolumn{7}{|l|}{ Lung } \\
\hline Yes & $3891(29.8)$ & $2388(28.1)$ & $752(34.1)$ & $442(31.3)$ & 309 (32.6) & $<0.001$ \\
\hline No & $8636(66.1)$ & $5749(67.6)$ & $1386(62.9)$ & $902(64.0)$ & $599(63.1)$ & \\
\hline Unknown & $539(4.1)$ & $366(4.3)$ & $66(3.0)$ & $66(4.7)$ & $41(4.3)$ & \\
\hline \multicolumn{7}{|l|}{ Follow-up duration in months } \\
\hline Mean $(95 \% \mathrm{CI})$ & $22.6(22.3-23.0)$ & $23.0(22.6-23.4)$ & $20.1(19.4-20.8)$ & $23.9(23.0-24.9)$ & $23.5(22.3-24.6)$ & \\
\hline Median (IQR) & $19.0(8.0-34.0)$ & $20.0(7.0-35.0)$ & $17.0(6.0-30.0)$ & $20.0(10.0-35.0)$ & $20.0(8.0-36.0)$ & \\
\hline \multicolumn{7}{|l|}{ Follow-up status ${ }^{c}$} \\
\hline Alive & $5340(40.9)$ & $3521(41.4)$ & $735(33.3)$ & $636(45.1)$ & $448(47.2)$ & $<0.001$ \\
\hline Dead due to breast cancer & 6999 (53.6) & $4507(53.0)$ & $1328(60.3)$ & $706(50.1)$ & $458(48.3)$ & $<0.001$ \\
\hline Dead due to other causes & $727(5.6)$ & $475(5.6)$ & $141(6.4)$ & $68(4.8)$ & $43(4.5)$ & 0.097 \\
\hline
\end{tabular}

$C I$ confidence interval, $H E R 2$ human epidermal growth factor receptor-2, $H o R$ hormone receptor, $I D C$ invasive ductal carcinoma, $I L C$ invasive lobular carcinoma, $I Q R$ interquartile range, $N H A$ non-Hispanic Asian or Pacific Islander/American Indian/Alaska Native, $N H B$ non-Hispanic black, $N H W$ non-Hispanic white, $Q$ quintile, $S E S$ socioeconomic status, $U D$ undifferentiated

${ }^{a} P$ value was assessed using the Pearson's $\chi^{2}$

${ }^{\mathrm{b}}$ Including divorced, separated, single (never married), and widowed

${ }^{\mathrm{c}}$ As of December 31, 2017

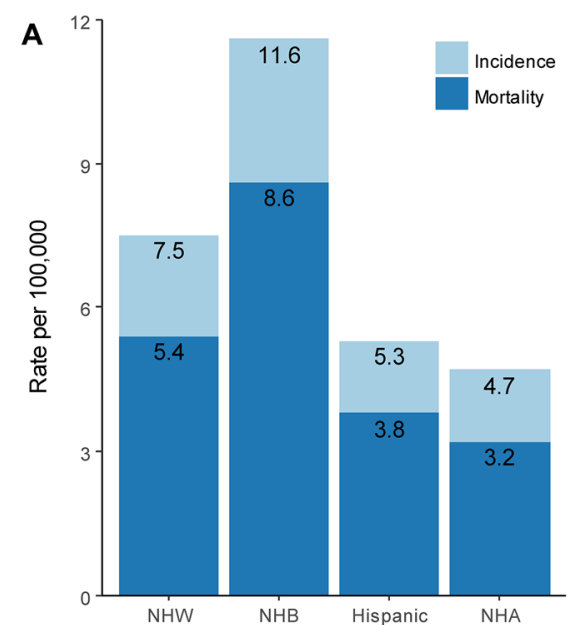

Fig. 2 Incidence of metastatic breast cancer and incidence-based mortality rates (2010-2014) and age-specific rates (2010-2014) in female patients stratified by race/ethnicity. The rates were adjusted for age based on the US standard population in 2000. NHA non-Hispanic
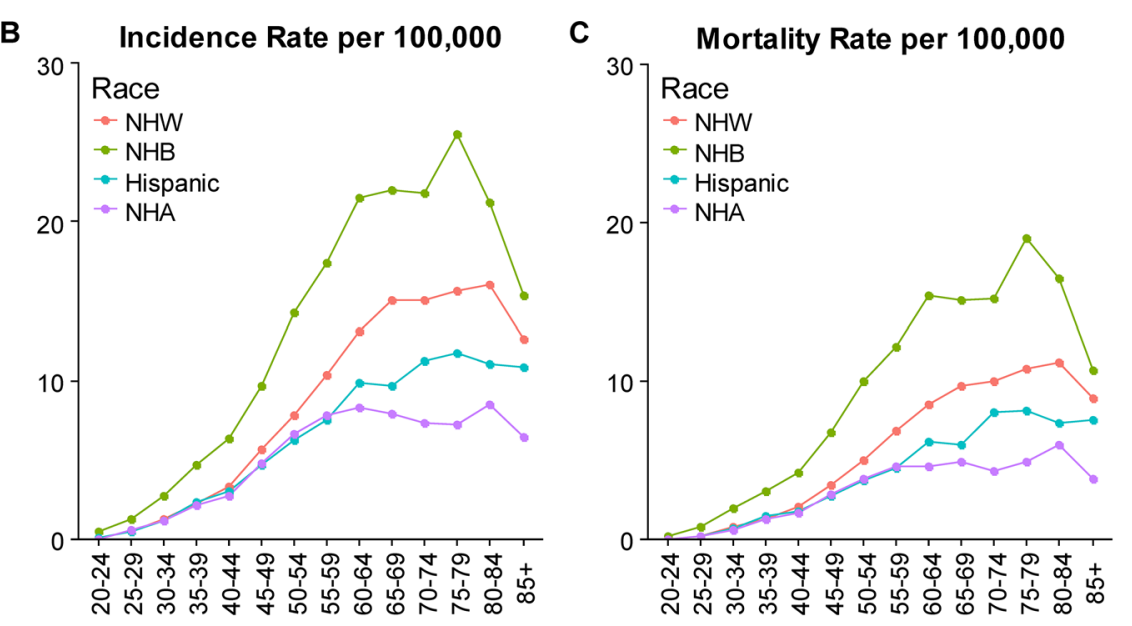

Asian or Pacific Islander/American Indian/Alaskan Native, $N H B$ nonHispanic black, $N H W$ non-Hispanic white. Sources North American Association of Central Cancer Registries, 2017 
64.4\%) and the human epidermal growth factor receptor-2 (HER2)-/HoR - subtype (18.9\% vs. 10.9\%). NHW women were the most likely group to be diagnosed with smallersized tumors (15.8\%), the HER2-/HoR+ subtype (54.9\%), a single distant metastasis $(53.3 \%)$, or bone metastasis $(67.3 \%)$.

\section{Differences in the outcomes of groups stratified by race/ethnicity}

Univariate and multivariate proportional hazards models for both OS and BCSS were employed to determine prognostic factors for patients with metastatic breast cancer (Tables 2 , $\mathrm{S} 1)$. The death risk was substantially higher in NHB women compared with that in NHW women, and this risk was mitigated in the fully adjusted model (HR for BCSS, 1.24 vs. 1.07; HR for OS, 1.27 vs. 1.12). Hispanic women exhibited a better survival rate in both the univariate and multivariate analyses and the prognosis of NHA women was not significant in the multivariate model. Additionally, after adjusting for all the listed prognostic factors in the multivariate analysis, patients who were diagnosed at an older age with worse socioeconomic support (uninsured, unmarried, and poor SES neighborhoods), advanced tumor characteristics (grade III/UD, tumor size $>50 \mathrm{~mm}$, and HER2-/HoR- subtype), and known specific organ metastases (bone/brain/liver/lung) were at an increased risk of death. Patients with a young onset, a higher SES and the HER2+/HoR + subtype showed a decreased risk of death $(P<0.05)$.

HRs for cancer-specific death between NHB and NHW women were attenuated after adjusting for socioeconomic, tumor or metastatic characteristics, but these HRs were increased in age-adjusted models. These factors together accounted for $69.5 \%$ of the total excess risk of death in NHB women compared with that in NHW women. In the respective adjusted models, the estimated proportion of excess risk attributed to socioeconomic factors was $66.5 \%$, followed by tumor characteristics at $41.5 \%$, and the metastatic pattern at $14.8 \%$. Conversely, with the imbalance of the age distribution, the racial differences were aggravated by $40.3 \%$ (Table 3).

Table 3 further lists HRs for death resulting from breast cancer, in NHB and NHW women stratified by age. Among patients who were diagnosed with metastatic breast cancer at a younger age (18-49 years), NHB women were $57.0 \%$ more likely to die than NHW women (HR 1.57; 95\% CI $1.37-1.80$ ) and the excess risk was decreased to $13.4 \%$ (HR 1.13 ; 95\% CI 0.96-1.34) after multivariate adjustment. These factors together accounted for $76.5 \%$ of the total excess risk; in the respective adjusted models, the excess risk attributed to socioeconomic factors was $51.4 \%$, followed by tumor characteristics at $40.7 \%$, and the metastatic pattern at $15.8 \%$. Among middle-aged patients (50-64 years),
NHB women were $42.2 \%$ more likely to die than NHW women (HR 1.42; 95\% CI 1.30-1.56), and the excess risk decreased to $10.8 \%$ (HR 1.11; 95\% CI 1.00-1.23) in the fully adjusted model. These factors together accounted for $74.4 \%$ of the total excess risk; the excess risk mediated by socioeconomic factors was $53.6 \%$, followed by tumor characteristics at $33.9 \%$, and the metastatic pattern at $3.1 \%$. Among the elderly group (aged $65+$ years), no racial difference in breast cancer-specific survival was observed (HR 1.10; 95\% CI 0.99-1.22). Table S2 lists the HRs for BCSS in NHW and NHB women with narrowed age categories, excluding the 18-34 age group, due to an insufficient number of events and lack of statistical significance. Consistent with the above analysis, the excess mortality risk of NHB women was increased significantly in the younger age group (aged $<45$ years) and non-significantly among the elderly group (aged $65+$ years). The results were similar for total mortality (Table S3).

\section{Subgroup analysis of NHW and NHB women}

After adjustment for all listed prognostic factors in the model of BCSS, prognostic differences in NHB and NHW women persisted in less aggressive subgroups with higherSES individuals and neighborhoods, tumors at the T2 stage $(21-50 \mathrm{~mm})$ with the ductal histological type, and a lack of existing brain metastases (Fig. 3).

\section{Discussion}

Population-based estimates of the racial disparities in metastatic breast cancer are lacking. By providing a comprehensive assessment of racial differences in the incidence and outcomes of patients with de novo metastatic breast cancer, this retrospective study confirmed and extended the higher risk of NHB women coupled with a large population, a precise definition of subtype and detailed information on metastatic sites. The two dominant contributors to these racial differences were socioeconomic factors and tumor characteristics, the latter of which further explained the strengthened racial disparities in survival among the younger group. Furthermore, white-black survival disparities persisted in less aggressive subgroups, underscoring the importance of implementing more intensive strategies for detecting and treating metastases in non-elderly NHB women with better SES, tumors at the T2 stage with the ductal histological type, and a lack of existing brain metastases.

According to national epidemiologic data $[1,2,18]$, the overall incidence of breast cancer (2005-2014) is higher in NHW women in the US; however, for metastatic breast cancer, NHB women showed a higher incidence in the present study. A decreasing trend in the mortality rate (2005-2014) 
Table 2 Univariate and multivariate Fine and Gray model analyses for breast cancer-specific mortality

\begin{tabular}{|c|c|c|c|c|c|c|}
\hline & \multirow[t]{2}{*}{ No. of patients } & \multirow[t]{2}{*}{ No. of deaths } & \multicolumn{2}{|l|}{ Univariate analysis } & \multicolumn{2}{|c|}{ Multivariate analysis } \\
\hline & & & $\mathrm{CIF}(95 \% \mathrm{CI})$ & $P$ & CIF $(95 \% \mathrm{CI})$ & $P$ \\
\hline \multicolumn{7}{|l|}{ Race } \\
\hline NHW & 8503 & 4507 & Reference & - & Reference & - \\
\hline NHB & 2204 & 1328 & $1.24(1.16-1.31)$ & $<0.001$ & $1.07(1.00-1.15)$ & 0.047 \\
\hline Hispanic & 1410 & 706 & $0.92(0.85-0.99)$ & 0.034 & $0.91(0.84-0.99)$ & 0.029 \\
\hline NHA & 949 & 458 & $0.91(0.83-0.99)$ & 0.035 & $0.94(0.85-1.04)$ & 0.250 \\
\hline \multicolumn{7}{|c|}{ Age at diagnosis, years } \\
\hline 1-year increase & & & $1.01(1.01-1.02)$ & $<0.001$ & $1.02(1.01-1.02)$ & $<0.001$ \\
\hline \multicolumn{7}{|l|}{ Insurance status } \\
\hline Insured & 9539 & 4951 & Reference & - & Reference & - \\
\hline Medicaid & 2636 & 1522 & $1.19(1.13-1.26)$ & $<0.001$ & $1.17(1.10-1.25)$ & $<0.001$ \\
\hline Uninsured & 891 & 526 & $1.35(1.23-1.48)$ & $<0.001$ & $1.30(1.18-1.44)$ & $<0.001$ \\
\hline \multicolumn{7}{|l|}{ Marital status } \\
\hline Married & 5552 & 2689 & Reference & - & Reference & - \\
\hline Not married ${ }^{\mathrm{a}}$ & 6751 & 3902 & $1.35(1.29-1.42)$ & $<0.001$ & $1.16(1.10-1.22)$ & $<0.001$ \\
\hline \multicolumn{7}{|c|}{ Neighborhood SES } \\
\hline Q1 (low) & 2515 & 1473 & Reference & - & Reference & - \\
\hline Q2 & 2624 & 1457 & $0.93(0.87-1.00)$ & 0.062 & $0.94(0.87-1.01)$ & 0.110 \\
\hline Q3 & 2747 & 1480 & $0.88(0.82-0.95)$ & $<0.001$ & $0.90(0.84-0.97)$ & 0.007 \\
\hline Q4 & 2596 & 1341 & $0.80(0.75-0.87)$ & $<0.001$ & $0.86(0.79-0.93)$ & $<0.001$ \\
\hline Q5 (high) & 2419 & 1169 & $0.73(0.67-0.78)$ & $<0.001$ & $0.80(0.74-0.87)$ & $<0.001$ \\
\hline \multicolumn{7}{|l|}{ Histological type } \\
\hline IDC & 8782 & 4570 & Reference & - & Reference & - \\
\hline ILC & 1372 & 721 & $0.98(0.91-1.05)$ & 0.560 & $1.12(1.03-1.22)$ & 0.011 \\
\hline Others & 2912 & 1708 & $1.25(1.18-1.32)$ & $<0.001$ & $1.09(1.02-1.16)$ & 0.007 \\
\hline \multicolumn{7}{|l|}{ Grade } \\
\hline $\mathrm{I} / \mathrm{II}$ & 5005 & 2300 & Reference & - & Reference & - \\
\hline III/UD & 5230 & 3062 & $1.46(1.38-1.53)$ & $<0.001$ & $1.35(1.27-1.43)$ & $<0.001$ \\
\hline \multicolumn{7}{|l|}{ Tumor size(mm) } \\
\hline $0-20$ & 1907 & 925 & Reference & - & Reference & - \\
\hline $21-50$ & 4743 & 2303 & $1.00(0.93-1.08)$ & 0.930 & $1.03(0.95-1.11)$ & 0.520 \\
\hline$>50$ & 3903 & 2194 & $1.27(1.18-1.37)$ & $<0.001$ & $1.20(1.11-1.30)$ & $<0.001$ \\
\hline \multicolumn{7}{|c|}{ Regional lymph nodes positive } \\
\hline No & 2860 & 1507 & Reference & - & Reference & - \\
\hline Yes & 8803 & 4573 & $0.94(0.89-1.00)$ & 0.035 & $0.96(0.90-1.02)$ & 0.170 \\
\hline \multicolumn{7}{|l|}{ Molecular subtype } \\
\hline HER2-/HoR+ & 6866 & 3389 & Reference & - & Reference & - \\
\hline HER $2+/ H o R+$ & 1930 & 828 & $0.84(0.78-0.90)$ & $<0.001$ & $0.77(0.71-0.83)$ & $<0.001$ \\
\hline HER $2+/ H o R-$ & 1056 & 501 & $1.02(0.93-1.12)$ & 0.660 & $0.90(0.81-0.99)$ & 0.028 \\
\hline HER2-/HoR- & 1612 & 1224 & $2.27(2.12-2.42)$ & $<0.001$ & $2.04(1.89-2.20)$ & $<0.001$ \\
\hline \multicolumn{7}{|c|}{ No. of distant metastases } \\
\hline 1 & 6808 & 3224 & Reference & - & Reference & - \\
\hline$>1$ & 5356 & 3186 & $1.47(1.40-1.55)$ & $<0.001$ & $1.09(1.03-1.15)$ & 0.004 \\
\hline \multicolumn{7}{|l|}{ Bone } \\
\hline Yes & 8515 & 4583 & $1.02(0.97-1.08)$ & 0.380 & $1.23(1.16-1.30)$ & $<0.001$ \\
\hline No & 4245 & 2217 & Reference & - & Reference & - \\
\hline \multicolumn{7}{|l|}{ Brain } \\
\hline Yes & 938 & 6567 & $2.23(2.06-2.42)$ & $<0.001$ & $1.96(1.79-2.13)$ & $<0.001$ \\
\hline No & 11,567 & 5926 & Reference & - & Reference & - \\
\hline
\end{tabular}


Table 2 (continued)

\begin{tabular}{|c|c|c|c|c|c|c|}
\hline & \multirow[t]{2}{*}{ No. of patients } & \multirow[t]{2}{*}{ No. of deaths } & \multicolumn{2}{|l|}{ Univariate analysis } & \multicolumn{2}{|c|}{ Multivariate analysis } \\
\hline & & & CIF (95\% CI) & $P$ & CIF $(95 \%$ CI) & $P$ \\
\hline \multicolumn{7}{|l|}{ Liver } \\
\hline Yes & 3412 & 2209 & $1.71(1.62-1.80)$ & $<0.001$ & $1.77(1.67-1.88)$ & $<0.001$ \\
\hline No & 9218 & 4513 & Reference & - & Reference & - \\
\hline \multicolumn{7}{|l|}{ Lung } \\
\hline Yes & 3891 & 2370 & $1.47(1.39-1.54)$ & $<0.001$ & $1.24(1.17-1.31)$ & $<0.001$ \\
\hline No & 8636 & 4277 & Reference & - & Reference & - \\
\hline
\end{tabular}

$C I$ confidence interval, HER2 human epidermal growth factor receptor-2, $H o R$ hormone receptor, $H R$ hazard ratio, IDC invasive ductal carcinoma, ILC invasive lobular carcinoma, NHA non-Hispanic Asian or Pacific Islander/American Indian/Alaska Native, $N H B$ non-Hispanic black, $N H W$ non-Hispanic white, $Q$ quintile, $S E S$ socioeconomic status, $U D$ undifferentiated

ancluding divorced, separated, single (never married), and widowed

for all-stage breast cancer but a stable trend for metastatic breast cancer were observed. Moreover, NHB women showed significantly higher morbidity and mortality of metastatic breast cancer at any age.

However, the extent of racial variances changed when the data were stratified by age. We found more substantial differences in both BCSS and OS in younger patients with metastatic breast cancer, whereas an excess risk of death was not observed among elderly NHB women. A study from Vaz et al. [6] focused on elderly patients with de novo metastatic breast cancer who were diagnosed between 1998 and 2009 supported a non-significant white-black difference in OS. Regarding racial disparities in survival among patients with all-stage breast cancer, Sighoko et al. [3] reported similar results for cohorts stratified by age in ten US cities with large African American populations; nevertheless, baseline characteristics other than age were not provided due to a lack of information. Taken together, these findings highlight the importance of age-based guidelines for breast cancer screening and the early detection of distant metastases and that these guidelines should consider race, particularly for non-elderly NHB women.

Differences in socioeconomic support have previously been cited as reasons for overall racial differences in breast cancer prognoses [7, 13, 19-21]. Low-SES individuals and neighborhoods at diagnosis were consistently associated with worse survival in NHB patients than in NHW patients with breast cancer [22-24]. We extended these conclusions to metastatic breast cancer. A low SES accounted for approximately one-half of the excess survival risk in NHB women compared with that in NHW women (BCSS: total, $66.5 \%$; $18-49$ years, $51.4 \%$; 50-64 years, $53.6 \%$; $65+$ years, NA; OS: total, $68.9 \%$; 18-49 years, 48.1\%; 50-64 years, $50.4 \%$; $65+$ years, NA). For the $65+$ age group, the racial disparity in risk was not significant, which could be partially explained by relatively uniform health care coverage through Medicare in this age group compared with other age cohorts. Therefore, for non-elderly patients with no comparable insurance coverage, equal access to care reduces racial/ ethnic disparities in prognosis.

Differences in tumor biology have also been consistently cited as contributors to racial/ethnic disparities in breast cancer mortality $[5,6,9,10,25-33]$. In the present study, this factor explained approximately two-fifths (41.5\%) of the total excess cancer-specific risk. This estimated percentage is larger than that found in previous studies (Warner et al. [34], 23.8\%; Jemal et al. [8], 25.8\%). However, direct comparison is limited by the differences in the analyzed populations (stage IV vs. stages I to III). Advanced tumor characteristics play a more important role in younger patients with metastatic breast cancer, as mentioned above (BCSS: 18-49 years, 40.7\% ; 50-64 years, 33.9\%; 65+ years, NA; OS: $18-49$ years, $35.1 \%$; 50-64 years, $24.9 \%$; $65+$ years, NA). These results reflect a higher percentage of patients diagnosed at a younger age $(<50$ years) among NHB women than among NHW women $(26.7 \%$ vs. $16.3 \%)$, together with an even more disproportionate number of patients with the HER2-/HoR - subtype (20.0\% vs. $12.6 \%)$, larger tumor size ( $>50 \mathrm{~mm}, 36.5$ vs. 26.8 ), regional lymph node infiltration $(79.3 \%$ vs. $72.3 \%$ ), and other aggressive characteristics associated with a poorer survival among young NHB women (Table S4). A similar pattern was observed among patients diagnosed at a median age (50-64 years), who presented smaller disparities, but not among patients aged $65+$. BRCA1/2 gene mutations, particularly the rapidly progressive and highly malignant HER2-/HoR - subtype, are reported to closely correlate with the aggressive young-onset phenotype in NHB women [35-38]. With advances in breast cancer genomics, more comprehensive studies are expected to clarify potential genome-based differences.

Differences in the metastatic pattern explained approximately one-tenth of the excess risk (BCSS: $14.8 \%$; OS: $12.0 \%)$, which was narrowed but not eradicated after stratification by age (18-49 years, $15.8 \%$; 50-64 years, 3.1\%; 


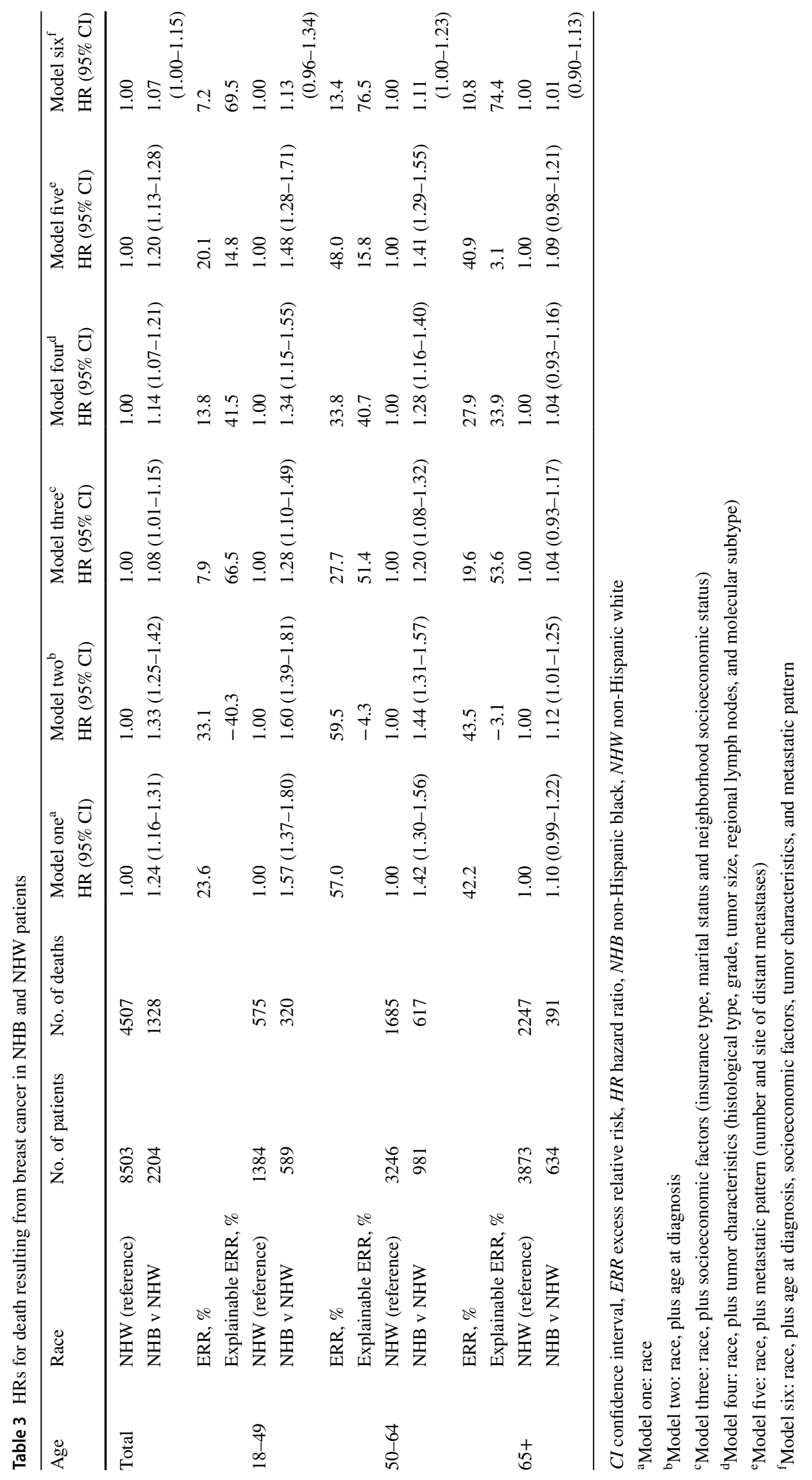




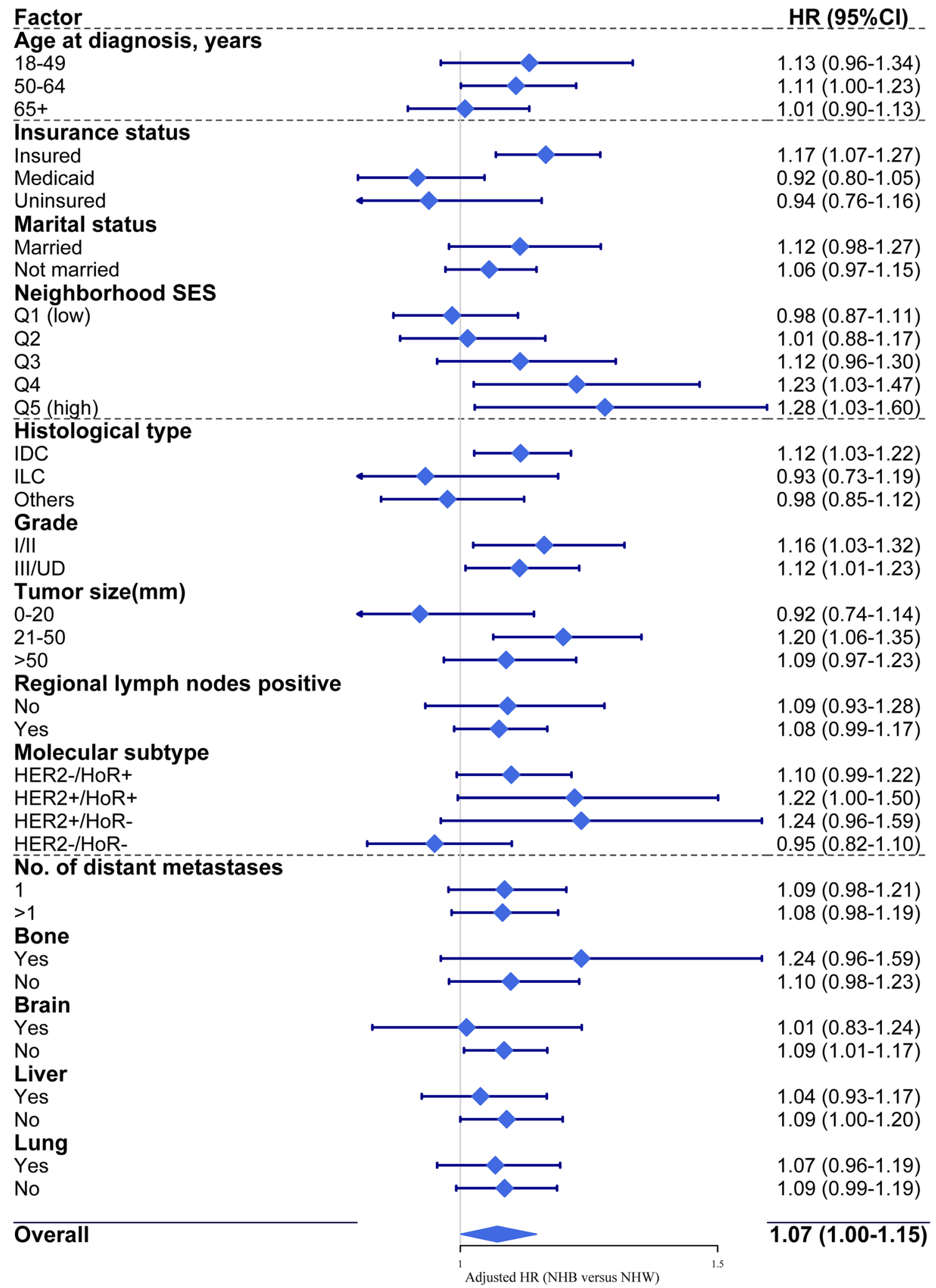

Fig. 3 Forest plot showing the results of the multivariate proportional hazards model analysis of breast cancer-specific mortality in NHB and NHW women stratified by subgroup after adjustment for all other prognostic factors listed. The diamond denotes the HR of each subgroup. An HR $>1.0$ indicates a higher risk of breast cancer-specific mortality in NHB women. $C I$ confidence interval, HER2 human epidermal growth factor receptor-2, HoR hormone receptor, $H R$ hazard ratio, $I D C$ invasive ductal carcinoma, $I L C$ invasive lobular carcinoma, $N H B$ non-Hispanic black, $N H W$ non-Hispanic white, $Q$ quintile, $S E S$ socioeconomic status, $U D$ undifferentiated 
$65+$ years, NA). The higher proportion of bone-only metastases in NHW women accounted for the substantially better survival [39-42]. In comparison, NHB women exhibited more brain metastases, which were potentially correlated with the higher percentage of the HoR-negative subtype [28, 43].

NHB women presented an absolute disparity in the rate of suffering more aggressive tumors, and a relative disparity in white-black survival also persisted in less aggressive subgroups. Similar results were observed based on SES among all-stage breast cancer and other solid malignancies [9, 21-23]. We extended the conclusion to more comprehensive prognostic factors including demographic, socioeconomic, tumor and metastatic characteristics. Insured NHB patients from high-SES neighborhoods, exhibiting tumors at the T2 stage with the ductal histological type, and without existing brain metastases suffered poor survival compared with their NHW counterparts, suggesting that NHW patients obtained greater benefits from improved access to care. Some possible explanations for this disparity include differences in the accessibility and utilization of and compliance with systemic treatments such as endocrine therapy, HER2-targeted therapy or chemotherapy due to patient-level social, cultural, linguistic, or even financial barriers [6, 44]. Large populationbased studies involving more detailed explorations of how treatment patterns affect outcomes among races/ethnicities are warranted in the future. Another potential explanation includes delayed detection resulting from asymptomatic distant metastases among NHB women with seemingly better prognostic factors. Brain MRI for early detection of brain metastases is recommended by Martin et al. [43] for patients with the HER2+/HoR- and HER2-/HoR- subtypes, which comprise more than one-quarter of NHB patients. Efforts to reduce lagging survival among NHB women with metastatic breast cancer are needed in both less and more aggressive groups.

For Hispanics and NHAs, relatively better survival was observed in our study, together with a younger onset and lower SES. These findings warrant additional attention to the civil awareness of breast cancer, disparities in access to care and potential genetic susceptibility. Both the Hispanic and NHA groups are significantly heterogenetic racial/ethnic groups, comprising subgroups that differ in country of origin, primary language, and geographic location. Thus, the survival of Hispanic or NHA patients with metastatic breast cancer varies with the distribution of prognostic factors in each study population [45-48]. Further comparisons among specific subgroups were limited by the small sample size of the available data and warrant future studies.

\section{Limitations}

Our study has several limitations. First, although the NCI SEER database is the largest cancer database in the United States, it still does not cover $100 \%$ of the US population, with different percentages of coverage being observed according to race/ethnicity. Nevertheless, the heterogeneity of the population included in this database, coupled with its large sample size, supports the validity of our findings. Additionally, the results presented here are specific to the population distribution in the United States, and caution should be taken in generalization to other geographic regions/ countries. Second, information about disease recurrence or subsequent sites involved is not available in the SEER database; thus, we were only able to include de novo metastases rather than relapsed metastatic cases. Future investigations using alternative data sources to include relapsed metastases are warranted. Third, because NCCN guidelines do not recommend routine systemic staging for patients with asymptomatic early-stage breast cancer, underestimation of the incidence of de novo metastatic breast cancer could exist. Fourth, the age-adjusted mortality rates calculated in the present study were not directly based on mortality data collected by the National Center for Health Statistics (1969-2015) because partitioning of mortality by associated variables is permitted only in the Incidence-based Mortality database (2000-2014). Fifth, other potential socioeconomic factors, such as occupation, education, income, and tax, were not examined because patient-level information is not available in the SEER database. Finally, we cannot comment on comorbidities, body mass indexes, family histories, genomic mutations, treatment patterns or toxicities due to a lack of such information.

\section{Conclusions}

Despite all these limitations, our study provides insights into the racial variations in the incidence and outcomes of de novo metastatic breast cancer using recent data obtained from 13,066 women across the United States. As an explanation for the distribution of excess risk for different factors computed in the age-stratified groups, we identified lower survival of non-elderly NHB women than of NHW women, urging the implementation of targeted preventive efforts in public health, policy, and clinical arenas. Furthermore, clinicians should focus more on non-elderly NHB patients in less aggressive subgroups who are more likely to suffer distant metastases than their NHW counterparts. We urge multidimensional verification of and potential intervention in survival disparities in the clinical field. 
Acknowledgements We thank SEER for providing open access to the database. We thank American Journal Experts (AJE) for English language editing. We thank all of the subjects who were included in this study for their participation.

Author contributions ZMS, XH, HL, YG, and JXR conceived and designed the study. YG and JXR calculated the statistics and analyzed the data. JXR prepared the figures and tables and wrote the main manuscript. ZMS and XH supervised the entire project. All of the authors reviewed the manuscript.

Funding Support was provided by the Ministry of Science and Technology of China (National Key R\&D Program of China, MOST2016YFC0900300), the National Natural Science Foundation of China $(81572583,81672601,81602311)$ and the Shanghai Committee of Science and Technology Funds (15410724000). The funders had no role in the study design, collection and analysis of the data, decision to publish, or manuscript preparation.

\section{Compliance with ethical standards}

Conflict of interest The authors declare that they have no conflict of interest.

Ethical approval This study was conducted with the approval of the Ethical Committee Review Board of Fudan University Shanghai Cancer Center and did not include human participants; therefore, informed consent was not required.

Open Access This article is distributed under the terms of the Creative Commons Attribution 4.0 International License (http://creativeco mmons.org/licenses/by/4.0/), which permits unrestricted use, distribution, and reproduction in any medium, provided you give appropriate credit to the original author(s) and the source, provide a link to the Creative Commons license, and indicate if changes were made.

\section{References}

1. DeSantis CE, Ma J, Goding Sauer A et al (2017) Breast cancer statistics, 2017, racial disparity in mortality by state. CA 67:439448. https://doi.org/10.3322/caac.21412

2. DeSantis CE, Fedewa SA, Goding Sauer A et al (2016) Breast cancer statistics, 2015: convergence of incidence rates between black and white women. CA 66:31-42. https://doi.org/10.3322/ caac. 21320

3. Sighoko D, Hunt BR, Irizarry B et al (2018) Disparity in breast cancer mortality by age and geography in 10 racially diverse US cities. Cancer Epidemiol 53:178-183. https://doi.org/10.1016/j. canep.2018.02.003

4. Stapleton SM, Oseni TO, Bababekov YJ et al (2018) Race/ ethnicity and age distribution of breast cancer diagnosis in the United States. JAMA Surg. https://doi.org/10.1001/jamas urg.2018.0035

5. Arciero CA, Yang J, Peng L et al (2017) African American patients with breast cancer have worse prognosis than white patients in certain subtypes and stages. Breast Cancer Res Treat 166:743-755. https://doi.org/10.1007/s10549-017-4484-1

6. Vaz-Luis I, Lin NU, Keating NL et al (2015) Racial differences in outcomes for patients with metastatic breast cancer by disease subtype. Breast Cancer Res Treat 151:697-707. https://doi. org/10.1007/s10549-015-3432-1

7. Singh GK, Jemal A (2017) Socioeconomic and racial/ethnic disparities in cancer mortality, incidence, and survival in the United States, 1950-2014: over six decades of changing patterns and widening inequalities. J Environ Public Health 138:1-19. https:// doi.org/10.1155/2017/2819372

8. Jemal A, Robbins AS, Lin CC et al (2018) Factors that contributed to black-white disparities in survival among nonelderly women with breast cancer between 2004 and 2013. J Clin Oncol 36:14 24. https://doi.org/10.1200/JCO.2017.73.7932

9. Ellis L, Canchola AJ, Spiegel D et al (2017) Racial and ethnic disparities in cancer survival: the contribution of tumor, sociodemographic, institutional, and neighborhood characteristics. J Clin Oncol. https://doi.org/10.1200/JCO.2017.74.2049

10. Daly B, Olopade OI (2015) A perfect storm: how tumor biology, genomics, and health care delivery patterns collide to create a racial survival disparity in breast cancer and proposed interventions for change. CA 65:221-238. https://doi.org/10.3322/ caac. 21271

11. Surveillance Research Program, National Cancer Institute SEER*Stat software (http://www.seer.cancer.gov/seerstat) version 8.3 .5

12. Surveillance, Epidemiology, and End Results (SEER) Program (2017) (http://www.seer.cancer.gov) SEER*stat database: incidence-SEER 18 Regs excluding AK (with additional treatment fields), Nov 2017 Sub (2000-2015) < Vintage 2015 pops by race/ origin tract 2000/2010 mixed geographies $>$-linked to census tract attributes-time dependent (2000-2015) - SEER 18 (excl AK) Census 2000/2010 Geographies with Index Field Quantiles, National Cancer Institute, DCCPS, Surveillance Research Program, released June 2018, based on the November 2017 submission.

13. Kish JK, Yu M, Percy-Laurry A (2014) Racial and ethnic disparities in cancer survival by neighborhood socioeconomic status in Surveillance, Epidemiology, and End Results (SEER) Registries. J Natl Cancer Inst 2014:236-243. https://doi.org/10.1093/jncim onographs/lgu020

14. Yost K, Perkins C, Cohen R et al (2001) Socioeconomic status and breast cancer incidence in California for different race/ethnic groups. Cancer Causes Control 12:703-711

15. Surveillance, Epidemiology, and End Results (SEER) Program (2018) (http://www.seer.cancer.gov) SEER*stat database: incidence-based mortality-SEER 18 Regs (Excl Louisiana) Research Data, Nov 2017 Sub (2000-2015) < Katrina/Rita Population Adjustment $>$ - Linked to County attributes - total U.S., 19692016 Counties, National Cancer Institute, DCCPS, Surveillance Research Program, released April 2018, based on the November 2017 submission.

16. Dignam JJ, Zhang Q (2012) The use and interpretation of competing risks regression models. Clin Cancer Res 18:2301-2308. https ://doi.org/10.1158/1078-0432.CCR-11-2097

17. Fine JP, Gray RJ (1999) A proportional hazards model for the subdistribution of a competing risk. J Am Stat Assoc 94:496-509

18. DeSantis CE, Siegel RL Sauer AG et al (2016) Cancer statistics for African Americans, 2016: progress and opportunities in reducing racial disparities. CA 66:290-308. https://doi.org/10.3322/ caac. 21340

19. Moss JL, Liu B, Feuer EJ (2017) Urban/rural differences in breast and cervical cancer incidence: the mediating roles of socioeconomic status and provider density. Women's Heal Issues 27:683691. https://doi.org/10.1016/j.whi.2017.09.008

20. Yin D, Morris C, Allen M et al (2010) Does socioeconomic disparity in cancer incidence vary across racial/ethnic groups? Cancer Causes Control 21:1721-1730. https://doi.org/10.1007/s1055 2-010-9601-y 
21. Komenaka IK, Martinez ME, Pennington RE Jr et al (2010) Race and ethnicity and breast cancer outcomes in an underinsured population. J Natl Cancer Inst 102:1178-1187. https://doi. org/10.1093/jnci/djq215

22. Pan H, Walker G, Grant $\mathrm{S}$ et al (2017) Insurance status and racial disparities in cancer-specific mortality in the United States: a population-based analysis. Cancer Epidemiol Biomark Prev 26:869-875. https://doi.org/10.1158/1055-9965.EPI-16-0976

23. DeRouen MC, Parsons HM, Kent EE et al (2017) Sociodemographic disparities in survival for adolescents and young adults with cancer differ by health insurance status. Cancer Causes Control 28:841-851. https://doi.org/10.1007/s10552-017-0914-y

24. Martínez ME, Anderson K, Murphy JD et al (2016) Differences in marital status and mortality by race/ethnicity and nativity among California cancer patients. Cancer 122:1570-1578. https://doi. org/10.1002/cncr.29886

25. Carey LA, Perou CM, Livasy CA et al (2006) Race, breast cancer subtypes, and survival in the Carolina breast cancer study. JAMA 295:2492-2502. https://doi.org/10.1001/jama.295.21.2492

26. Tao L, Chu L, Wang L et al (2016) Occurrence and outcome of de novo metastatic breast cancer by subtype in a large, diverse population. Cancer Causes Control 27:1127-1138. https://doi. org/10.1007/s10552-016-0791-9

27. Keegan TH, DeRouen MC, Press DJ et al (2012) Occurrence of breast cancer subtypes in adolescent and young adult women. Breast Cancer Res 14:1-9. https://doi.org/10.1186/bcr3156

28. Anders D, Deal AM, Miller CR et al (2011) The prognostic contribution of clinical breast cancer subtype, age, and race among patients with breast cancer brain metastases. Cancer 117:16021611. https://doi.org/10.1002/cncr.25746

29. Sposto R, Keegan TH, Vigen C et al (2016) The effect of patient and contextual characteristics on racial/ethnic disparity in breast cancer mortality. Cancer Epidemiol Biomark Prev 25:1064-1072. https://doi.org/10.1158/1055-9965.EPI-15-1326

30. Iqbal J, Ginsburg O, Rochon PA et al (2015) Differences in breast cancer stage at diagnosis and cancer-specific survival by race and ethnicity in the United States. JAMA 313:165-173. https://doi. org/10.1001/jama.2014.17322

31. Ning J, Peng S, Ueno N et al (2015) Has racial difference in causespecific death improved in older patients with late-stage breast cancer? Ann Oncol 26:2161-2168. https://doi.org/10.1093/annon $\mathrm{c} / \mathrm{mdv} 330$

32. Shoemaker ML, White MC, Wu M et al (2018) Differences in breast cancer incidence among young women aged 20-49 years by stage and tumor characteristics, age, race, and ethnicity, 20042013. Breast Cancer Res Treat. https://doi.org/10.1007/s1054 9-018-4699-9

33. Tao L, Gomez SL, Keegan TH et al (2015) Breast cancer mortality in african-american and non-hispanic white women by molecular subtype and stage at diagnosis: a population-based study. Cancer Epidemiol Biomark Prev 24:1039-1045. https:// doi.org/10.1158/1055-9965.EPI-15-0243

34. Warner ET, Tamimi RM, Hughes ME et al (2015) Racial and ethnic differences in breast cancer survival: mediating effect of tumor characteristics and sociodemographic and treatment factors. J Clin Oncol 33:2254-2261. https://doi.org/10.1200/JCO.2014.57.1349
35. Davis MB, Newman LA (2018) Breast cancer disparities how can we leverage genomics to improve outcomes? Surg Oncol Clin N Am 27:217-234. https://doi.org/10.1016/j.soc.2017.07.009

36. Rummel L, Shriver E (2017) Contribution of germline mutations in cancer predisposition genes to tumor etiology in young women diagnosed with invasive breast cancer. Breast Cancer Res Treat 164:593-601. https://doi.org/10.1007/s10549-017-4291-8

37. Parada H, Sun X, Fleming JM et al (2017) Race-associated biological differences among luminal A and basal-like breast cancers in the Carolina Breast Cancer Study. Breast Cancer Res 19:131. https://doi.org/10.1186/s13058-017-0914-6

38. Churpek JE, Walsh T, Zheng Y et al (2015) Inherited predisposition to breast cancer among African American women. Breast Cancer Res Treat 149:31-39. https://doi.org/10.1007/s1054 9-014-3195-0

39. Xiong Z, Deng G, Huang X et al (2018) Bone metastasis pattern in initial metastatic breast cancer: a population-based study. Cancer Manag Res 10:287-295. https://doi.org/10.2147/CMAR.S155524

40. Parkes A, Clifton K, Al-Awadhi A et al (2018) Characterization of bone only metastasis patients with respect to tumor subtypes. npj Breast Cancer 4:2. https://doi.org/10.1038/s41523-018-0054-X

41. Leone BA, Vallejo CT, Romero AO et al (2017) Prognostic impact of metastatic pattern in stage IV breast cancer at initial diagnosis. Breast Cancer Res Treat 161:537-548. https://doi.org/10.1007/ s10549-016-4066-7

42. Lee SJ, Park S, Ahn HK et al (2011) Implications of bone-only metastases in breast cancer: favorable preference with excellent outcomes of hormone receptor positive breast cancer. Cancer Res Treat 43:89-95. https://doi.org/10.4143/crt.2011.43.2.89

43. Martin AM, Cagney DN, Catalano PJ et al (2017) Brain metastases in newly diagnosed breast cancer: a population-based study. JAMA Oncol. https://doi.org/10.1001/jamaoncol.2017.0001

44. Kast K, Schoffer O, Link T et al (2017) Trastuzumab and survival of patients with metastatic breast cancer. Arch Gynecol Obstet 296:303-312. https://doi.org/10.1007/s00404-017-4421-x

45. Gomez S, Behren J, McKinley M et al (2017) Breast cancer in Asian Americans in California, 1988-2013: increasing incidence trends and recent data on breast cancer subtypes. Breast Cancer Res Treat 164:139-147. https://doi.org/10.1007/s1054 9-017-4229-1

46. Torre LA, Sauer AM, Chen MS Jr et al (2016) Cancer statistics for Asian Americans, Native Hawaiians. 2016: converging incidence in males and females. CA 66:182-202. https://doi.org/10.3322/ caac. 21335

47. Gomez SL, Glaser SL, Horn-Ross PL et al (2014) Cancer research in Asian American, native Hawaiian, and Pacific Islander populations: accelerating cancer knowledge by acknowledging and leveraging heterogeneity. Cancer Epidemiol Biomark Prev 23:22022205. https://doi.org/10.1158/1055-9965.EPI-14-0624

48. Banegas L (2012) Breast cancer characteristics and outcomes among Hispanic Black and Hispanic White women. Breast Cancer Res Treat 134:1297-1304. https://doi.org/10.1007/s1054 9-012-2142-1 